\title{
Mechanical Noise Enhances Signal Transmission in the Bullfrog Sacculus
}

\author{
Andrew A. Indresano, Jonathan E. Frank, Pamela Middleton, and Fernán Jaramillo \\ Department of Biology, Carleton College, Northfield, MN 55057, USA
}

Received: 27 August 2002; Accepted: 7 January 2003; Online publication: 14 March 2003

\section{ABSTRACT}

Noise has been commonly thought to degrade the performance of sensory systems. However, it is now clear that the detection and transmission of weak signals in sensory systems can be enhanced by noise via stochastic resonance (SR). In hair cells, the quality of mechanoelectrical transduction is enhanced up to twofold by nanometer level mechanical noise acting on the hair bundle. We wanted to know whether these gains could be preserved, perhaps even enhanced, as information flows across hair cell synapses, and into the stream of action potentials that in the frog conveys acoustic information to the central nervous system. To approach this question, we studied the effects of noise on the signal-to-noise ratio (SNR) of the 8th nerve's response to small mechanical stimuli directly applied to the amphibian sacculus. We found that $\sim 2.5 \mathrm{~nm}$ of mechanical noise enhanced the response of the saccular nerve up to fourfold, suggesting that the positive effects of lowamplitude mechanical noise result in improved transmission of acoustic information.

Keywords: hair cell, stochastic resonance, sacculus, acoustic, signal processing

\section{INTRODUCTION}

The sacculus in modern frogs (neobatrachians) is an organ of superb sensitivity, detecting groundborne vibrations as small as $10^{-6} \mathrm{G}$ (Lewis et al.

Correspondence to: Fernán Jaramillo • Department of Biology • Carleton College - Northfield, MN 55057-4025. Telephone: (507) 646-4392; fax: (507) 646-5757; email: fjaramil@carleton.edu
2001). (For a review on the acoustic periphery of amphibians, see Lewis and Narins 1999.) Like other sensory organs, the sacculus embodies a compromise between natural limitations and the ability of natural selection to produce refined designs. What are the natural constraints that limit the sensitivity of this and other sensory systems? Noise is the answer that has been traditionally offered (de Vries 1950; Harris 1968; Corey and Hudspeth 1983b). Thus, it has been popularly thought that there is no selective advantage in developing more sensitive systems because smaller signals, even if intrinsically detectable, would be drowned out by the unavoidable background noise that arises from external sources as well as that originating from the intrinsic operation of sensory receptors, afferent fibers, etc. (Gulick et al. 1997).

Although noise can limit sensory systems, a "noiselimited" view of sensation tends to ignore the effects of noise on the behavior of some nonlinear dynamic systems. These effects are manifested as stochastic resonance (SR) which can be described as a nonlinear cooperative effect whereby a system's optimal signal-to-noise ratio (SNR) occurs at a nonzero noise level. During the past decade a significant body of evidence has accumulated which documents the role of SR in biological systems (Wiesenfeld and Moss 1995; Wiesenfeld and Jaramillo 1998).

We demonstrated earlier that mechanical noise comparable to that of a hair bundle's Brownian motion could enhance the SNR of mechanoelectrical transduction in isolated hair cells from the bullfrog sacculus (Jaramillo and Wiesenfeld 1998). This enhancement (up to $3 \mathrm{~dB}$ ) can be explained by SR acting on the mechanoelectrical transduction channels. However, several other steps in the information chain are intrinsically nonlinear and, because they 
take place in the presence of considerable noise, they are potential candidates for SR.

Here we sought to establish whether mechanical noise acting on the sacculus could enhance the organ's overall performance in the bullfrog (Rana catesbeiana). To accomplish this aim we used a new preparation that allows the direct application of mechanical stimuli to the sacculus and the recording of afferent activity from the saccular branchlet of the 8th nerve. Tests designed to measure the effects of mechanical noise on the sacculus' performance were preceded by experiments that characterized the behavior of the preparation.

\section{METHODS}

\section{Preparation}

Animals were anesthetized in ice water, pithed, and decapitated. The dorsal side of the head was pinned, ventral side up, to the Sylgard (Dow Corning, Midland, MI) bottom of a glass recording chamber. The head was completely submerged in a "no-calcium" saline $[(\mathrm{mM}) 110 \mathrm{NaCl}, 2 \mathrm{KCl}, 1 \mathrm{EGTA}, 3$ glucose, 5 HEPES, pH 7.25 with $1 \mathrm{~N} \mathrm{NaOH}]$ saturated with oxygen. A scalpel was used to remove a thin slice of the otic capsule's ventral wall, creating a small opening $(\sim 2.5 \mathrm{~mm})$ that allowed access to the sacculus (Fig. 1A). In general, preparations were used for a period no longer than two hours after sacrificing the animal.

\section{Mechanical stimulation}

Mechanical stimuli were conveyed to the sacculus by a stiff glass probe, approximately $100 \mu \mathrm{m}$ in diameter, which was affixed to the extramacular area of the sacculus using cyanoacrylate glue (Isodent, Elmira International Inc., Hewlett, NY). To couple the probe to the sacculus, the probe's tip was dabbed with glue and then rapidly advanced (to prevent curing) through the fluid until it contacted the sacculus. The sacculus was displaced along the direction of the nerve fibers leaving the sacculus, a direction similar to that used for early experiments on the sacculus microphonic potential (Corey and Hudspeth 1983a). All displacements are given in nanometers $(\mathrm{nm})$ (root mean square).

Sinusoidal stimuli (typically $100 \mathrm{~Hz}$ ) were generated with a digital function generator (DS335, Stanford Research Systems, Sunnyvale, CA). Noise was generated with a second digital function generator under GPIB control (33120 Hewlett Packard, Palo Alto, CA). Noise was high-pass filtered at $1 \mathrm{~Hz}$ to remove small offsets, and low-pass filtered at $300 \mathrm{~Hz}(-3$ $\mathrm{dB}, 6 \mathrm{~dB}$ /octave) to remove high-frequency components. Signal and noise were added on a differential
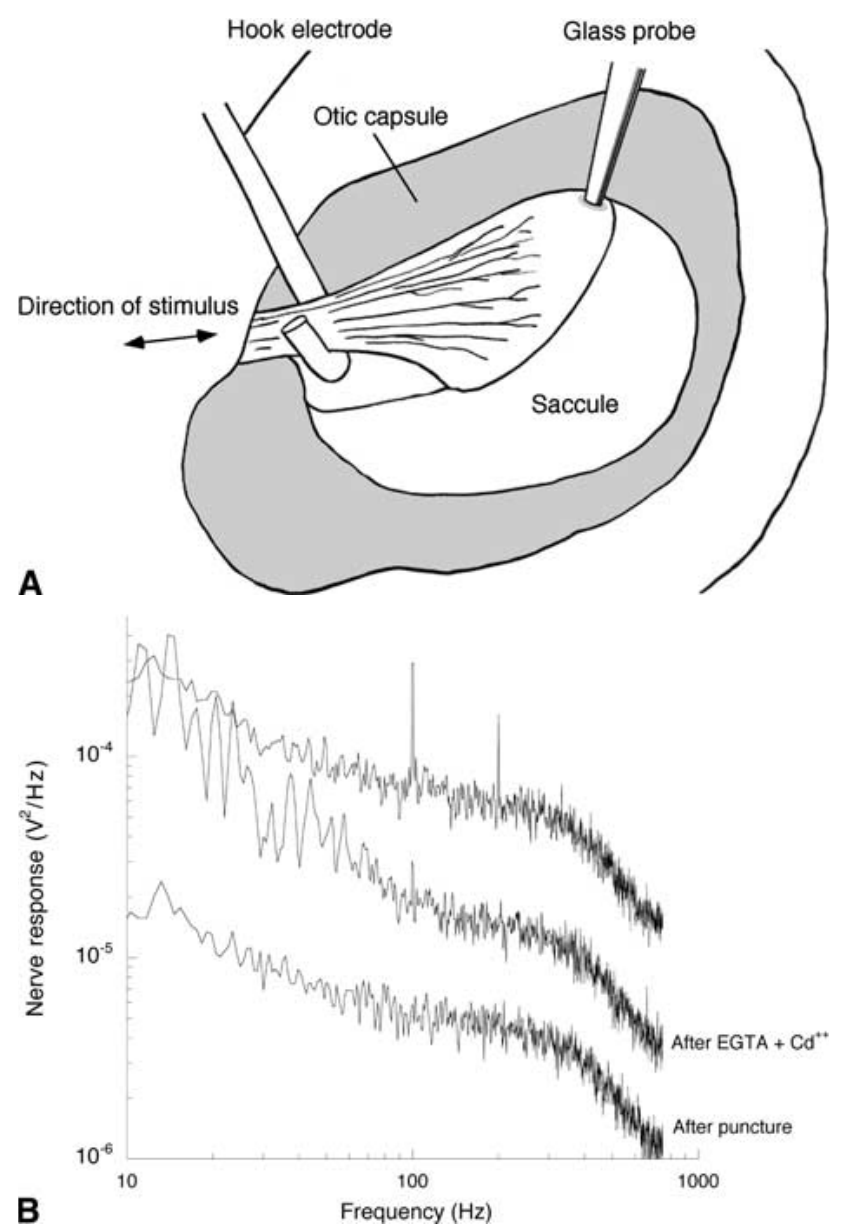

FIG. 1. Preparation and typical electrophysiological responses. A. Saccular preparation. A small opening was made in the otic capsule allowing access to the saccular nerve. A glass probe glued to the sacculus was used to stimulate the organ and the afferent nerve activity was registered using a chlorided silver electrode hooked around the nerve. B. Power spectra of nerve responses, showing the $f$ and $2 f$ components of nerve activity. The top spectrum was obtained while the sacculus was bathed in a perilymph-like solution. The middle spectrum was obtained immediately after the bath was replaced with our no-calcium saline (which contains 1 mM EGTA) supplemented with $100 \mu \mathrm{M} \mathrm{CdCl}_{2}$. The bottom spectrum was obtained in the same no-calcium saline immediately after the sacculus was gently punctured with a pair of fine forceps. To avoid overlap the two bottom spectra were divided by 30 and 100, respectively. The sacculus was displaced $28 \mathrm{~nm}$ at $100 \mathrm{~Hz}$. For clarity, peaks at 180 and $300 \mathrm{~Hz}$ were digitally removed. (The $180 \mathrm{~Hz}$ peak is a multiple of the line frequency. The $300 \mathrm{~Hz}$ peak is also a multiple of the line frequency, but it could also have a contribution from the saccular $3 f$ component).

amplifier, whose output was used to drive a closedloop piezoelectric stage (Queensgate, Devon, England) that controlled the position of the stimulus glass probe. To test the response of the stage to mechanical noise, we imaged a glass probe on a dual photodiode system as described elsewhere (Jaramillo and Hudspeth 1993). The SNR of the optically determined probe motion, as a function of noise added to the piezoelectric stimulator, declined monotoni- 
cally, ruling out nonlinearities in stimulation as a possible source of SR. Similarly, the power spectrum of probe fluctuations, when the probe was subjected to a small periodic stimulus, showed a component of motion only at the frequency of the stimulus. Peaks at harmonics of the primary frequency were undetectable.

To reduce extraneous mechanical noise the preparation was placed on a vibration isolation table (Technical Manufacturing, Peabody, MA) standing on a concrete platform about $3.5 \mathrm{~m}$ square separated by a $2.5 \mathrm{~cm}$ gap on its sides from the building's concrete floor, and enclosed by a $-40 \mathrm{~dB}$ attenuation sound booth (Industrial Acoustics, Bronx, NY). Although we did not monitor the probe's background noise during our experiments, we measured the residual mechanical noise at the surface of the table using a dual-photodiode system (Jaramillo and Wiesenfeld 1998; Frank et al. 2002). Residual noise was $0.5-0.75 \mathrm{~nm}$ in the $1.0 \mathrm{kHz}$ bandwidth.

\section{Stimulus presentation}

Usually a randomized presentation of stimuli is used to prevent history-dependent artifacts, which can be a legitimate concern. However, the vestibular and auditory systems are remarkably free of this type of artifact. Mechanoelectrical transduction is, within normal experimental limits, a memoryless process (adaptation of mechanoelectrical transduction can be nearly discounted at $100 \mathrm{~Hz}$ ). Similarly, hair cell synapses act in a way which is remarkably free of timedependent effects (Hind et al. 1966a, 1966b). Moreover, saccular nerve fibers show little adaptation, which in other systems may dull the effects of noise (Henry 1999).

Consider, in contrast, a SNR that is independent of added mechanical noise (i.e., no stochastic resonance) but that declines gradually (though occasionally rapidly) as the preparation fatigues. Since the SNR is independent of noise, the randomization of the presentation according to noise level would in effect randomize the abscissa. Such randomization would produce an artifactual peak in the SNR in the majority of cases, which would be highly undesirable. In contrast, the sequential presentation of noise levels tends to obscure the presence of SR peaks, which allows us to make conservative claims. Therefore, noise levels were presented in an increasing amplitude sequence.

\section{Recording}

Dissection was performed in a no-Ca ${ }^{2+}$ saline to prevent synaptic transmission, thus preventing the preparation from fatiguing (see Results). After the silver electrode and glass probe were in place, this no$\mathrm{Ca}^{2+}$ saline was replaced with a solution containing perilymph-like levels of $\mathrm{Ca}^{2+}[(\mathrm{mM}) 110 \mathrm{NaCl}, 2 \mathrm{KCl}$, $4 \mathrm{CaCl}_{2}, 3$ glucose, 5 HEPES, pH 7.25 with $1 \mathrm{~N}$ $\mathrm{NaOH}]$. Compound afferent activity was monitored with a chlorided silver wire wrapped around the saccular branchlet of the 8th nerve, using a high-inputimpedance differential AC amplifier (Model 1700, AM Systems, Carlsborg, WA). The difference signal between the nerve and bath electrodes was amplified 10,000-fold, high-pass filtered at $10 \mathrm{~Hz}$ to remove drift, low-pass filtered at $500 \mathrm{~Hz}$, and monitored using a dual-phase-sensitive-detector lock-in amplifier (SR830, Stanford Research Systems). The lock-in amplifier was locked to the sync output of the sinusoidal stimulus function generator. To estimate the amplitude $(V)$ of the differential amplifier's signal at the stimulus frequency, we simultaneously measured the in-phase $(X=V \cos \mathrm{P})$ and quadrature $(Y=V$ $\sin \mathrm{P}$ ) components of the signal. The amplitude of the signal was then estimated as the square root of $X^{2}+Y^{2}$. This procedure allowed us to measure the sacculus response to sinusoidal stimuli of any amplitude and frequency, without regard to the phase delay of the response.

\section{Data acquisition and analysis}

The lock-in amplifier outputs were digitized at 1.0 $\mathrm{kHz}$ with a 16-bit E-series board (National Instruments, Austin, TX), operating under LabView ${ }^{\mathrm{TM}}$. This sampling rate is sufficient to prevent any aliasing, given the lock-in amplifier time constants (typically $1 \mathrm{~s}$ ). Signals were monitored for time intervals varying between 1 and $30 \mathrm{~s}$ (a constant value was used for any one set of noise values tested). For spectral analysis (e.g., Fig. 1B), the output of the differential AC amplifier was digitized at $1.5 \mathrm{kHz}$. Blocks of 1024 samples were collected and subjected to Fourier analysis. Spectra from 6 to 64 data segments were averaged to improve resolution. Data for the SNR vs. noise relationships were obtained with an automated routine in which the added mechanical noise was controlled by the computer via GPIB communication with the function generator. Following a change in the added noise level, the computer paused ( 5 time constants) for the lock-in output to reach its new steady-state amplitude. The output of the differential and lock-in amplifiers were sampled synchronously. The amplitude of the signal (usually at $100 \mathrm{~Hz}$ ) was estimated from the lock-in amplifier samples. To estimate the noise, the differential amplifier samples were windowed (Hanning), the power spectrum was computed and rescaled to correct for windowing, and the average noise level in a narrow band (typically 5 $\mathrm{Hz}$ ) centered at $94 \mathrm{~Hz}$ was estimated. For every 
preparation an additional measure of noise was obtained from the standard deviation of the lock-in amplifier's output, after correction for the amplifier's equivalent noise bandwidth. This estimate of noise includes contributions not only from the background, but from fluctuations in the signal as well. Similar SNR vs. noise plots were obtained with both estimates of noise, but all plots shown were obtained using the spectral noise estimate. To compare SNR plots obtained with different peak stimuli, or with different sacculi, each plot was normalized to the SNR obtained in the absence of added mechanical noise (this also tends to correct for definitions of noise which are intrinsically arbitrary). Thus, the ordinate in all SNR plots (Figs. 3 and 4) starts at 1.

\section{RESULTS}

\section{Response of the preparation to periodic stimuli}

The response of the saccular nerve to the sinusoidal displacement of the sacculus at a frequency $f$ exhibited prominent peaks at the primary $(f)$ and second harmonic (2f) frequencies (Fig. 1B, top spectrum). Several factors are likely to contribute to components at all harmonics of the primary frequency: the nonlinear displacement response relationship of mechanoelectrical transduction, the presence of two populations of hair cells oriented in opposite directions across the striola, and the nonlinear behavior of firing neurons. Although the relative amplitude of the $f$ and $2 f$ peaks varied considerably for different preparations, the amplitude of the first peak was consistently the largest, and therefore we focused on this component in our experiments. To determine that these peaks resulted from nerve activity, we tested the effects on the preparation of conditions known to interfere with either synaptic transmission or nerve activity: Both peaks were reversibly suppressed (90-100\%) by the addition to the bath of 0.1 $\mathrm{M} \mathrm{CdCl}_{2}$ (not shown), by the replacement of the normal $\mathrm{Ca}^{2+}$ bath with the no-Ca ${ }^{2+}$ saline (see Methods), or by a combination of both (Fig 1B, middle spectrum). The peaks were also abolished by $1 \mu \mathrm{M}$ tetrodotoxin, or by $20 \mathrm{mM} \mathrm{KCl}$, both of which interfere with nerve activity, the former by blocking $\mathrm{Na}^{+}$ channels, and the latter by depolarizing the nerve fibers, thus driving $\mathrm{Na}^{+}$channels into the inactivated sate. In general, all peaks disappeared entirely when the nerve was intentionally damaged by gently rubbing it with the recording electrode. Occasionally, a small fraction of the $f$ component remained after blocking synaptic transmission or nerve activity (Fig $1 \mathrm{~B}$, middle spectrum). We attributed this residual peak to a contribution to the $f$ component by the mechanoelectrical transduction currents flowing

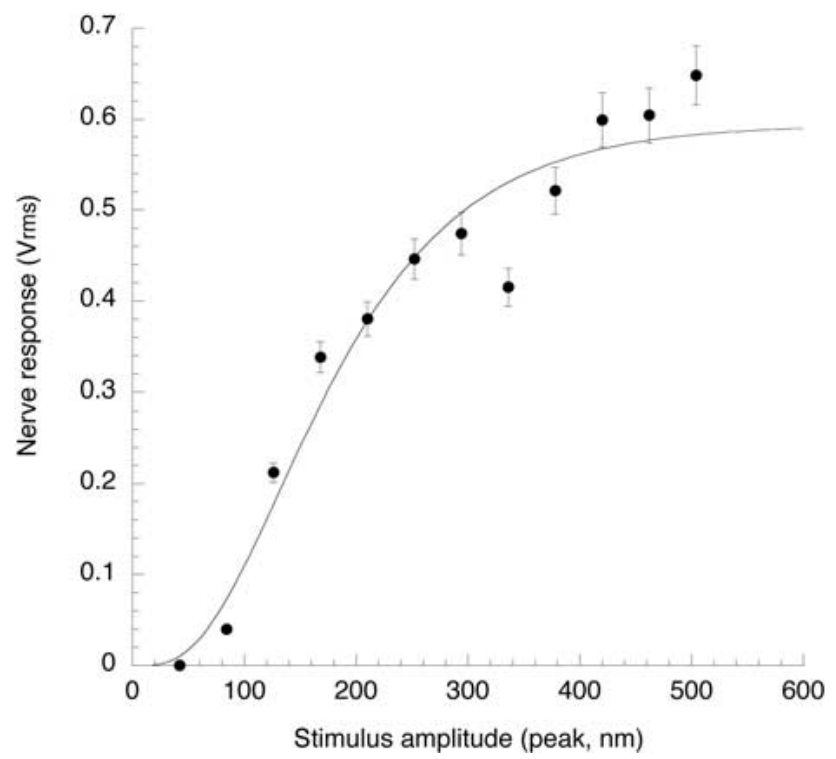

FIG. 2. Displacement-response relationship for an individual sacculus. The stimuli consisted of $100 \mathrm{~Hz}$ sine waves of varying amplitude. The stimuli were applied in order of increasing amplitude for a period of $4 \mathrm{~s}$ and the signal detected by the lock-in amplifier was simultaneously recorded. Error bars are standard deviation. The data were fitted to the fourth order sigmoidal curve $Y=A \cdot\left(1-e^{-X / B}\right)^{4}$, which is used only as a rough guide. The relationship was obtained automatically using a computer-controlled routine and a constant low sensitivity in the lock-in amplifier to prevent saturation at the larger stimuli. At this sensitivity, the responses to smaller stimuli are poorly resolved and it appears that there was no response for stimuli below $60 \mathrm{~nm}$. However, at high sensitivity we observed well-behaved stimulus-response relationships for all stimuli used (not shown).

around the sacculus. All peaks disappeared completely following puncture of the sacculus with a fine probe (Fig. 1B, bottom spectrum).

\section{Frequency and amplitude dependence of nerve activity}

In preliminary experiments we removed the membranous labyrinth and tested its responsiveness in vitro. The only significant difference we detected with respect to recordings of the nerve in situ was the much more rapid deterioration of the preparation in vitro. The decline of the preparation in these preliminary in vitro experiments was consistently delayed by the addition of $0.1 \mathrm{M} \mathrm{CdCl}_{2}$ to the bath, suggesting a failure in transmitter release as the main reason for this deterioration. Deterioration occurred more rapidly as the frequency of stimulation was increased.

For both types of preparation the response of the nerve increased monotonically with the amplitude of the stimulus (Fig. 2). This response approached saturation for stimuli above $350 \mathrm{~nm}$, a value consistent with the saturation of the microphonic potential reported for bullfrog sacculi in response to direct dis- 


\section{DISCUSSION}

During the past decade much has been learned about the positive role that noise can play in sensory systems via SR. In has been reasonably well established that noise can enhance detection in the nervous system for both periodic and aperiodic signals (Collins et al. 1996; Jaramillo and Wiesenfeld 1998; Manjarrez et al. 2002). Furthermore, SR has also been shown to enhance neuronal information encoding (measured in terms of Shannon's transinformation rate) (Levin and Miller 1996). Our aim here was to inquire into what has not been thoroughly proven: first, that physiological levels of endogenous noise in the vestibular/auditory system lead to SR; second, that gains obtained at one stage of the sensory chain are maintained as information flows centrally.

We observed that, as our preparations deteriorated, the positive effects of noise vanished, though a detectable neural response could still be obtained. Several steps in the organ's response probably weaken with time. The ability of $\mathrm{Cd}^{2+}$ to slow down this decline suggests that a depletion of the hair cells' neurotransmitter plays a major role in a preparation's decay (mechanoelectrical transduction is rather sturdy and can be preserved for hours, even in a dissected sacculus, although a steady decline can also be observed). Thus, as a preparation declines, a larger displacement is required to elicit the same response from the nerve. These large displacements produce deterministic shifts in the open probability of the transduction channels. Such large shifts would be insensitive to modulation by random bundle fluctuations, explaining the disappearance of the SR effect. A similar explanation accounts for the lack of a SR effect for large stimuli in healthy preparations.

Our experiments indicate that the addition of a few nanometers of noise to a periodic stimulus leads to a substantial improvement ( $4 \mathrm{~dB}$, on average) in the SNR of the nerve's response. Such a dependence on noise could be important because this noise level is close to the Brownian motion experienced by unrestrained hair bundles (Denk et al. 1989, 1992). Our findings in the sacculus are potentially relevant to other mechanosensory systems. For example, hair bundles in cochlear inner hair cells may be uncoupled from the tectorial membrane and, therefore, their Brownian motion may enhance the sensitivity of mechanoelectrical transduction (Lindeman et al. 1971; Dallos and Harris 1978; Dallos 1985; Jaramillo and Wiesenfeld 1998). For most biological systems that exhibit SR, it is not precisely known what the naturally occurring noise levels are. Thus, SR improvements in sensation, which in most cases depend on the presence of an optimal noise level, may not occur naturally (Collins et al. 1995).
Direct evidence for the positive role of noise in acoustic and vestibular function has been reported in the literature (Yu and Lewis 1989; Morse and Evans 1996; Henry 1999). There are several possible mechanisms through which the SNR of afferent activity in hair cell organs may be enhanced. Here we list four notable ones, without attempting to quantify precisely the extent to which they account for our observations. The first mechanism involves a depolarizing DC bias, either in the hair cells or in their afferent fibers. This effect enhances the organs' response by bringing the afferent synaptic potential closer to the threshold they might otherwise not reach (Lowenstein 1956). In nerve fibers this mechanism is likely to be effective only in the short term, unless the resting membrane potential is relatively hyperpolarized, i.e., not within the range of $\mathrm{Na}^{+}$channel activation (otherwise $\mathrm{Na}^{+}$ channel inactivation is likely to reduce activity, as seen in accommodating fibers). There is also a possibility that at higher frequencies of stimulation the resulting DC bias in the hair cell membrane potential would result in an increased level of transmitter release. In general, we do not think that DC bias plays a significant role in our results. The second mechanism involves the effect of noise in linearizing spike initiation in afferent fibers, thus allowing the nerve fiber to respond by modulating its firing rate continuously in response to a varying input (French and Stein 1970; $\mathrm{Yu}$ and Lewis 1989). This is analogous to dithering, a widely used digitization technique in which one trades off distortion in the digitization of small signals for noise, which can later be removed by filtering. The third mechanism involves an SR effect on the likelihood that the synaptic potential reaches threshold. In this case noise acts as an instantaneous bias on the spike initiation mechanism. Finally, noise can act on the hair bundle via SR, which can be viewed as a dynamic version of dithering, to optimize the SNR of mechanoelectrical transduction (Jaramillo and Wiesenfeld 1998). There is also a possibility that noise might enhance other nonlinear processes in the chain of communication between the hair bundle and afferent firing, such as the gating of voltage-dependent $\mathrm{Ca}^{2+}$ channels and the release of neurotransmitter.

The observed gains in the response of the saccular nerve are substantial when compared with the overall dynamic range of the organ (about $12 \mathrm{~dB}$ in Fig. 2), but note that at the $\mathrm{BF}$ dynamic range can exceed 60 $\mathrm{dB}$ in the bullfrog sacculus (Lewis et al. 2001). However, the key issue here is detection, and therefore a comparison with the dynamic range of the organ is somewhat misleading. For near-threshold signals a two- to fourfold increase in sensitivity may represent the difference between successful detection and becoming someone else's next dinner. Evolutionary pressure is on the side of increased sensitivity. In 
general, increased sensitivity is unlikely to create a "false alarm" problem because the response of the organism to sensory input can be modulated by factors such as alertness, fear, and fatigue. Noise could yield similar gains in the vestibular system; before this work was completed we received a report of similar results in the chick's semicircular canal (E. Manjarrez, University of Puebla, personal communication).

The observed improvement in the SNR of the nerve response occurs in response to rather small and narrowly constrained noise levels. Interestingly, the noise level that produces optimal SNRs in the nerve is virtually the same level that optimizes mechanoelectrical transduction, although the gains in the nerve appeared to exceed those observed for transduction (Jaramillo and Wiesenfeld 1998). This suggests that gains afforded by a given nonlinear process (e.g., transduction) can be preserved, and perhaps even enhanced at subsequent nonlinear steps in the sensory chain (e.g., synaptic transmission and action potential generation).

Two issues will require further investigation: first, are these noise amplitudes physiologically relevant in the sacculus where hair bundles are restrained by their association with the otoconial membrane? Second, how is the performance of the organ "tuned" to provide the relevant noise amplitudes? Evolution might have shaped the organ's response by taking advantage of other intrinsic sources of noise (e.g., membrane voltage noise). Experiments using individual fibers might offer insights which are obscured by en masse recordings. Moreover, although the SNR of the nerve's response is a valid measure of a sensory system's response, it is possible that other measures of performance, such as the coherence between action potentials in individual fibers, or the rate of information transfer, might provide a better insight into the role of noise in the saccule's function (Douglass et al. 1993; Collins et al. 1996; Levin and Miller 1996; Ward et al. 2002). The simple, yet rugged, preparation we have used here offers a convenient way to test some of these ideas.

\section{ACKNOWLEDGMENTS}

We thank K. Wiesenfeld for discussions. We also thank E. Manjarrez and his colleagues for discussions and for sharing their unpublished results. This work was supported by NIDCD grant DC02235-02.

\section{REFERENCES}

Collins JJ, Carson CC, Ihmoff TT. Stochastic resonance without tuning. Nature 376:236-238, 1995.
Collins JJ, Imhoff TT, Grigg P. Noise-enhanced information transmission in rat SA1 cutaneous mechanoreceptors via aperiodic stochastic resonance. J. Neurophys. 76:642-645, 1996.

Corey DP, Hudspeth AJ. Analysis of the microphonic potential of the bullfrog's sacculus. J. Neurosci. 3:942-961, 1983a.

COREY DP, HudSPETH AJ. Kinetics of the receptor current in bullfrog saccular hair cells. J. Neurosci. 3:962-976, 1983b.

DAllos P. Response characteristics of mammalian cochlear hair cells. J. Neurosci. 5:1591-1608, 1985.

Dallos P, Harris D. Properties of auditory nerve responses in absence of outer hair cells. J. Neurophys. 41:365-383, 1978.

DE VRIEs H. The mechanics of the labyrinth otoliths. Acta Otolaryngol. 38:262-273, 1950.

Denk W, Webi WW, Hudspeth AJ. Mechanical properties of sensory hair bundles are reflected in their Brownian motion measured with a laser differential interferometer. Proc. Natl. Acad. Sci. USA 86:5371-5375, 1989.

Denk W, Keolian RM, Webb WW. Mechanical response of frog saccular hair bundles to the aminoglycoside block of mechanoelectrical transduction. J. Neurophysiol. 68:927-932, 1992.

Douglass JK, Wilkens L, Pantazelou E, Moss F. Noise enhancement of information transfer in crayfish mechanoreceptors by stochastic resonance. Nature 365:337-340, 1993.

Frank JE, Markin V, Jaramillo F. Characterization of adaptation motors in saccular hair cells by fluctuation analysis. Biophys. J. 83:3188-3201, 2002.

French AS, Stein RB. A flexible neural analog using integrated circuits. IEEE Trans. Biomed. Eng. 17:248-253, 1970.

Gulick WL, Gescheider GA, Frisina RD. Hearing: Physiological Acoustics, Neural Coding, and Psychoacoustics, 2nd ed. Oxford University Press, New York, 1997.

HARRIS GG. Brownian motion in the cochlear partition. J. Acoust. Soc. Am. 44:176-186, 1968.

HeNRY KR. Noise improves transfer of near-threshold, phase-locked activity of the cochlear nerve: evidence for stochastic resonance? J. Comp. Physiol. A 184:577-584, 1999.

Hind JE, Anderson DJ, Brugge JF, Rose JE. Phase-locked response to low frequency tones in single auditory nerve fibers of the squirrel monkey. J. Neurophysiol. 30:794-793, 1966a.

Hind JE, Anderson DJ, Brugge JF, Rose JE. Coding of information pertaining to paired low-frequency tones in single auditory nerve fibers of the squirrel monkey. J. Neurophysiol. 30:794$816,1966 \mathrm{~b}$.

JaRAmillo F, Hudspeth AJ. Displacement-clamp measurement of forces exerted by gating springs in the hair bundle. Proc. Natl. Acad. Sci. USA 90:1330-1334, 1993.

JARAMILlo F, WIESENFELD K. Mechanoelectrical transduction assisted by Brownian motion: a role for noise in the auditory system. Nat. Neurosci. 1:384-388, 1998.

Koyama H, Lewis ER, Leverenz EL, Baird RA. Acute seismic sensitivity in the bullfrog ear. Brain Res. 250:168-172, 1982.

Levin JE, Miller JP. Broadband neural encoding in the cricket cereal sensory system enhanced by stochastic resonance. Nature 380:165-168, 1996.

Lewis ER. Tuning in the bullfrog ear. Biophys. J. 53:441-447, 1988.

LEWIS ER, NARINS PM. The acoustic periphery of amphibians: anatomy and physiology. In: FAY RR, Popper AN (eds) Comparative hearing: fish and amphibians. Springer-Verlag, New York, pp 101-154, 1999.

Lewis ER, Narins PM, Cortopassi KA, Yamada WM, Poinar EH, Moore SW, YU X-L. Do male white-lipped frogs use seismic signals for intraspecific communication? Am. Zool. 41:11851199, 2001.

Lindeman HH, Ades HW, Bredberg G, Engstrom H. The sensory hairs and the tectorial membrane in the development of the 
cat's organ of Corti. A scanning electron microscopic study. Acta Otolaryngol. 72:229-242, 1971.

Lowenstein O. Peripheral mechanisms of equilibrium. Br. Med. Bull. 12:114-118, 1956.

Manjarrez E, Diez-Martinez O, Mendez I, Flores A. Stochastic resonance in human electroencephalographic activity elicited by mechanical tactile stimuli. Neurosci. Lett. 324:213-216, 2002.

Morse RP, Evans EF. Enhancement of vowel coding for cochlear implants by addition of noise. Nat. Med. 2:928-932, 1996.
WARd LM, Neiman A, Moss F. Stochastic resonance in psychophysics and in animal behavior. Biol. Cybern. 87:91-101, 2002.

WIESENFELd K, Moss F. Stochastic resonance and the benefits of noise: from ice ages to crayfish and SQUIDs. Nature 373:33-36, 1995.

Wiesenfeld K, Jaramillo F. Minireview of stochastic resonance. Chaos 8:539-548, 1998.

Yu X, LEwIS ER. Studies with spike initiators: linearization by noise allows continuous signal modulation in neural networks. IEEE Trans. Biomed. Eng. 36:36-43, 1989. 